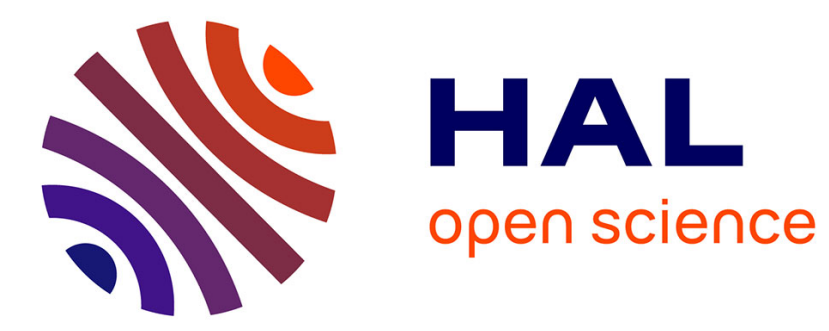

\title{
Modélisation du transducteur BF "Janus-Helmholtz". Nouvelle interprétation du fonctionnement et application à la tomographie acoustique de l'océan

\author{
Y. Le Gall
}

\section{- To cite this version:}

Y. Le Gall. Modélisation du transducteur BF "Janus-Helmholtz". Nouvelle interprétation du fonctionnement et application à la tomographie acoustique de l'océan. Journal de Physique IV Proceedings, 1994, 04 (C5), pp.C5-343-C5-346. 10.1051/jp4:1994571 . jpa-00253066

\section{HAL Id: jpa-00253066 https://hal.science/jpa-00253066}

Submitted on 1 Jan 1994

HAL is a multi-disciplinary open access archive for the deposit and dissemination of scientific research documents, whether they are published or not. The documents may come from teaching and research institutions in France or abroad, or from public or private research centers.
L'archive ouverte pluridisciplinaire HAL, est destinée au dépôt et à la diffusion de documents scientifiques de niveau recherche, publiés ou non, émanant des établissements d'enseignement et de recherche français ou étrangers, des laboratoires publics ou privés. 


\title{
Modélisation du transducteur BF "Janus-Helmholtz". Nouvelle interprétation du fonctionnement et application à la tomographie acoustique de l'océan
}

\author{
Y. LE GALL ${ }^{(1)}$ \\ IFREMER, Centre de Brest, Service DITI/SM/ASM, BP. 70, 29280 Plouzané, France
}

\begin{abstract}
Ocean Acoustic Tomography (measurement of the dynamic behaviour of the ocean) has needs for low frequency, high efficiency, broadband and great depth transducers, in order to reach ranges above $500 \mathrm{~km}$ [1]. The double-ended longitudinal "Janus-Helmholtz" projector designed by DCN INGENIERIE SUD widely answers these requirements. An attractive characteristic of such a transducer is the coupling between two resonances implying a large bandwidth.The first observed resonance is usually interpreted as a Helmholtz resonance and the second one as the driver resonance. First part of this study is about the working principles of the Janus-Helmholtz source. Two modelling tools have been used : the finite-element code ATILA and an equivalent circuit model. Thanks to them, a new interpretation of each resonance has been obtained and this transducer has then been optimized for acoustic tomography.
\end{abstract}

\section{INTRODUCTION : LE TRANSDUCTEUR DE TYPE JANUS-HELMHOLTZ}

\subsection{Description et performances}

L'utilisation des transducteurs Janus-Helmholtz comme sources basse fréquence constitue une solution innovante pour les applications océanographiques (tomographie acoustique) et sonar actif. Conçu par le département Lutte Sous-Marine de DCN INGENIERIE SUD, ce projecteur est composé d'un moteur piézoélectrique de type Janus, c'est à dire d'un pilier de céramiques inséré entre deux pavillons identiques (Fig, 1). Cette structure est montée à l'intérieur d'un baffle cylindrique ouvert en son milieu, créant ainsi l'évent de ce transducteur de type bass-reflex. Le découplage pavillons-boitier est assuré par une fente de quelques millimètres. Le baffle est alors tenu par la contremasse située au centre de l'ensemble qui correspond au noeud de vibration de la structure. L'utilisation de ce boîtier est nécessaire au bon fonctionnement du transducteur en empêchant les interactions entre les rayonnements avant et arrière des pavillons. De façon à satisfaire la condition de résonance de Helmholtz et pour couvrir une bande plus basse en fréquences, des tubes compliants remplis d'air sont insérés dans la cavité du projecteur.

L'utilisation de ce type de transducteur permet l'obtention de basses fréquences avec des niveaux élevés. La figure 2 représente la réponse à l'émission (SV) pour une tension d'excitation des céramiques de un Volt, dans le cas standard d'un Janus-Helmholtz avec dix tubes compliants. SV $0^{\circ}$ et SV $90^{\circ}$ correspondent respectivement au niveau dans l'axe du transducteur et au niveau radial.

Les expérimentations en tomographie acoustique nécessitant une largeur de bande supérieure à $100 \mathrm{~Hz}$, on s'intéresse à la réponse obtenue entre les deux résonances. La bande utile de situe alors autour de 700 $\mathrm{Hz}$ et le rendement électroacoustique très élevé dans cette bande (voisin de $80 \%$ ) est une caractéristique importante dans le cas de sources autonomes, permettant ainsi une minimisation de la puissance électrique.

Le problème de l'écrasement des tubes compliants avec la pression hydrostatique limitant alors l'immersion à $600 \mathrm{~m}$ (sans utilisation de système de compensation) a été résolu dans une étude parallèle [2].

\subsection{Intérêt de l'étude}

Jusqu'à présent, la première résonance, située à $600 \mathrm{~Hz}$ dans le cas du Janus-Helmholtz dix tubes, était assimilée à une résonance de Helmholtz, c'est à dire à un déplacement de fluide dans l'évent provoqué par le rayonnement arrière des pavillons dans la cavité compliante.

(1) Point de contact : DCN Ingénierie SUD/LSM, Le Brusc, 83140 Six-Fours-les-Plages, France 
La deuxième résonance, quant à elle, était associée à la résonance classique du moteur piézoélectrique, faisant intervenir sa masse dynamique et son élasticité. L'intérêt de cette étude est d'interpréter correctement chaque résonance, de façon à faire chuter les fréquences pour obtenir une bande située autour de $400 \mathrm{~Hz}$, indispensable pour les applications océanographiques longues portées.

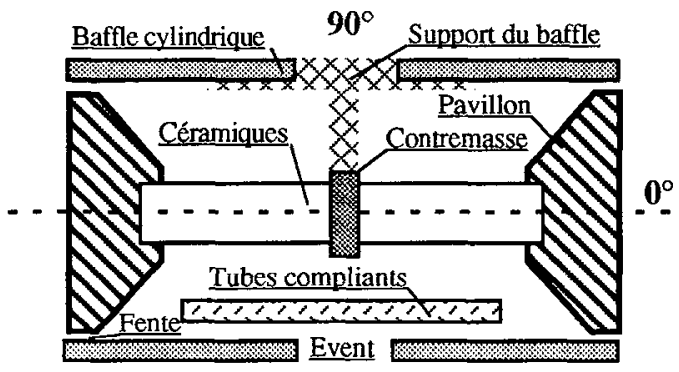

Fig. 1 : coupe longitudinale du Janus-Helmholtz

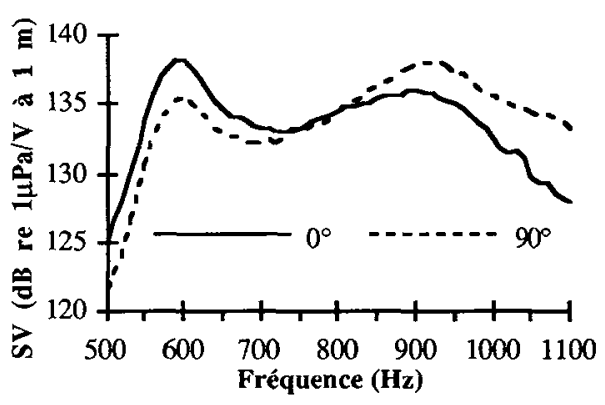

Fig. 2 : SV du Janus-Helmholtz 10 tubes

\section{OUTILS DE MODELISATION}

\subsection{Le code éléments-finis ATILA}

\subsubsection{Description du modele}

ATILA (Analyse de Transducteurs par Intégration des équations de LAplace) est un code éléments-finis spécialement développé pour permettre la modélisation de transducteurs rayonnant dans un fluide. Il peut être utilisé pour une analyse statique, modale ou harmonique de structures élastiques, piézoélectriques ou magnétostrictives [3].

Le Janus-Helmholtz est un transducteur à symétrie de révolution qui, de plus, présente un plan de symétrie : il est donc possible d'effectuer un calcul bidimensionnel axisymétrique. Ce maillage simplifie considérablement le modèle, mais n'autorise pas la prise en compte de la géométrie des tubes. La modélisation de ces éléments compliants est alors réalisée en homogénéisant leur effet sur l'ensemble du volume interne de la cavité, en excluant le volume de l'évent. La cavité est ainsi simulée comme un fluide de compressibilité équivalente à celle de l'eau ajoutée à celle des tubes.

Le caoutchouc de vulcanisation du transducteur est également modélisé et des pertes dans ce caoutchouc ainsi que dans le pilier de céramiques sont insérées sous la forme de matrices complexes.

\section{1 .2 Résultats}

Les figures 3 et 4 présentent la comparaison entre les résultats théoriques et expérimentaux dans l'axe radial (le transducteur étant disposé verticalement pour des besoins d'omnidirectionnalité en gisement, on s'intéresse principalement à la réponse dans cette direction) pour deux configurations de Janus-Helmholtz : sans tube et avec dix tubes.

Sans éléments compliants, l'accord entre les résultats expérimentaux et théoriques est bon en termes de fréquences et de niveaux. Les impédances électriques (non représentées) donnent également des résultats satisfaisants.

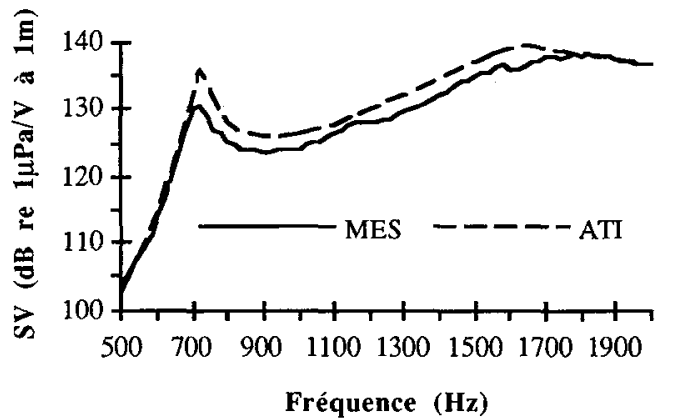

Fig. 3 : comparaison ATILA / Mesures (sans tube)

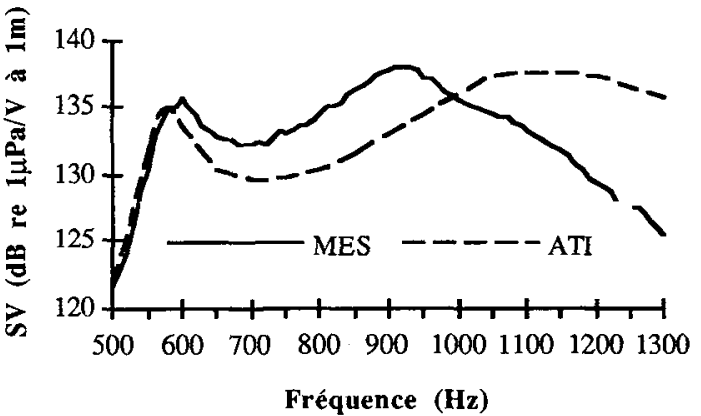

Fig. 4 : comparaison ATILA / Mesures (10 tubes) 
Dans le cas d'une cavité contenant des tubes, on constate un désaccord entre le modèle et la réalité pour la deuxième fréquence de résonance. Cet écart fréquentiel provient de la façon dont les tubes ont été simulés. En effet, une étude de la compliance d'un tube immergé (maillage tridimensionnel ATILA) a montré que cette dernière évoluait en fonction de la fréquence. La fréquence de résonance correspondant au mode symétrique de "respiration" du tube qui se situait au delà de $3 \mathrm{kHz}$ dans l'air se trouve à $1,4 \mathrm{kHz}$ dans l'eau. Un effet de "super compliance" se produit alors au voisinage de la deuxième résonance. Le remplacement de la compliance statique jusqu'alors utilisée par une compliance évoluant harmoniquement permettra alors de faire chuter la deuxième résonance.

\subsection{Les schémas à constantes localisées}

\subsubsection{Description et validité du modèle}

Un outil simple d'aide à la conception de transducteurs est donné par la méthode des schémas à constantes localisées. Le circuit électrique motionnel (parties mécanique et acoustique) équivalent du JanusHelmholtz est constitué de deux parties (Fig. 5) : le moteur et le résonateur (cavité et évent). Les différents termes sont des grandeurs acoustiques : dans cette analogie, la tension est équivalente à une pression et le courant à un débit.

Le moteur piézoélectrique (contremasse, pilier de céramiques, électrodes, tige de précontrainte et pavillons) est représenté par son élasticité $C_{\text {mot }}$, sa masse dynamique $M_{m o t}$, sa résistance de pertes $R_{m o t}$ et son impédance de rayonnement $Z_{\text {rpav }}\left(Z_{\text {rpav }}=R_{\text {rpav }}+j \omega M_{\text {rpav }}\right)$.

Le résonateur est modélisé par un circuit traduisant le phénomêne bass-reflex. La cavité est caractérisée par son élasticité $C_{c a v}$, sa résistance de pertes $R_{c a v}$ et la masse rajoutée sur la face arrière du pavillon

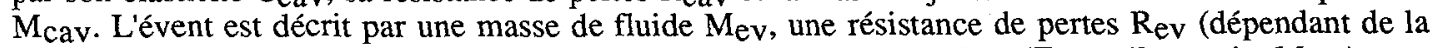
viscosité du fluide dans l'ouverture) et une impédance de rayonnement $Z_{r e v}\left(Z_{r e v}=R_{r e v}+j \omega M_{r e v}\right)$.

Compte-tenu du plan de symétrie de la structure, l'hypothèse adoptée pour le rayonnement des pavillons est celle de pistons plans circulaires dans un baffle infini. De plus, ce modèle à constantes localisées se limite au mode de compression-dilatation du moteur (vitesse uniforme sur une section perpendiculaire à la direction de déplacement), c'est à dire aux basses fréquences. Quant aux termes traduisant le rayonnement de l'évent cylindrique, ils sont déterminés à l'aide de la transformée de Hilbert qui, à partir de l'expression de la résistance de rayonnement de l'ouverture, permet de calculer sa masse acoustique de rayonnement (relations entre parties réelle et imaginaire).

\subsubsection{Résultats}

Pour obtenir un niveau radial (ou axial), chaque source (deux pavillons et un évent) a été considérée omnidirectionnelle et les divers déphasages ont été calculés permettant de tenir compte de chaque contribution (Fig. 6). Un décalage en fréquence est une nouvelle fois observé pour la deuxième résonance ; il pourra également être corrigé en intégrant dans le modèle l'évolution harmonique de la compliance des tubes.

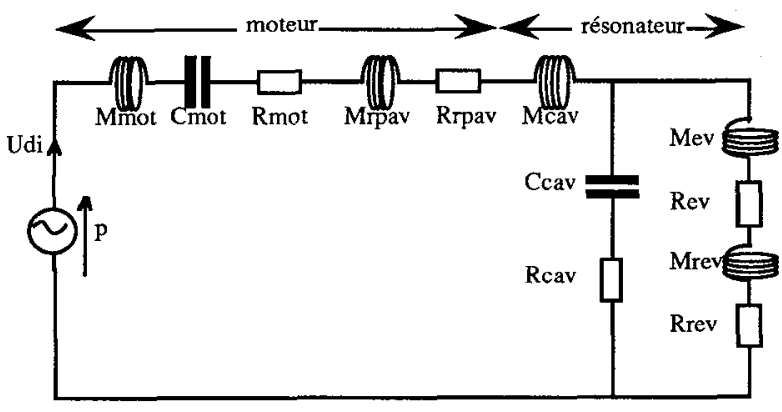

Fig. 5 : schéma électrique équivalent du transducteur Janus-Helmholtz

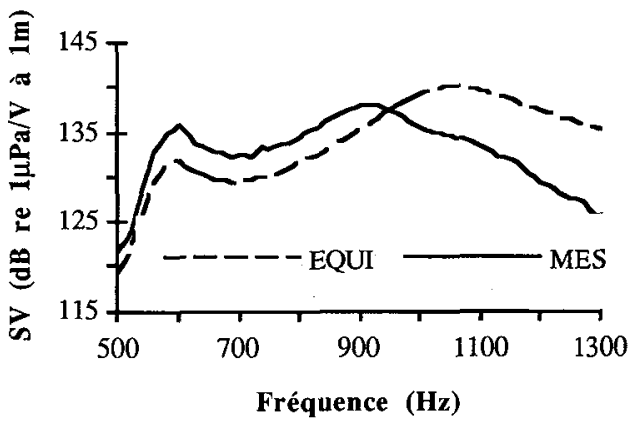

Fig. 6 : comparaison EQUI / Mesures (10 tubes)

\section{NOUVELLE INTERPRETATION DE CHAQUE RESONANCE}

\subsection{Effets de la compliance de la cavité et de la longueur du baffle}

Les réponses obtenues (mesures et modélisations) avec des Janus-Helmholtz possédant un nombre différent de tubes révèlent que l'élasticité de la cavité a beaucoup plus d'effet sur la deuxième résonance que sur la première (Fig. 7). Ce résultat est inattendu car il tend à contredire l'interprétation de la première résonance, jusqu'alors assimilée à une résonance de Helmholtz, résonance fortement dépendante de la compliance de la cavité. 
La modélisation de Janus-Helmholtz bafflés différemment indique que plus le baffle est long, plus la première résonance est basse en fréquence (Fig. 8). La décroissance régulière de la première résonance en fonction de la longueur du baffle tend à prouver qu'elle est de même nature que l'unique résonance d'un transducteur non bafflé. Cette dernière étant la résonance du moteur piézoélectrique, cela infirme la présence d'une résonance de Helmholtz à la fréquence correspondant à la première résonance.

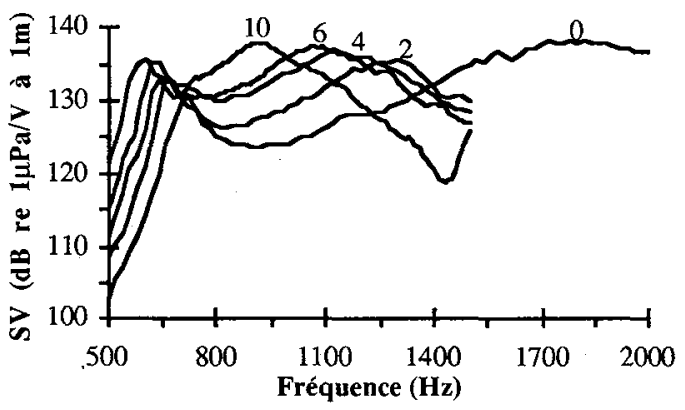

Fig. 7 : réponse radiale avec cavités différentes ( 0 à 10 tubes)

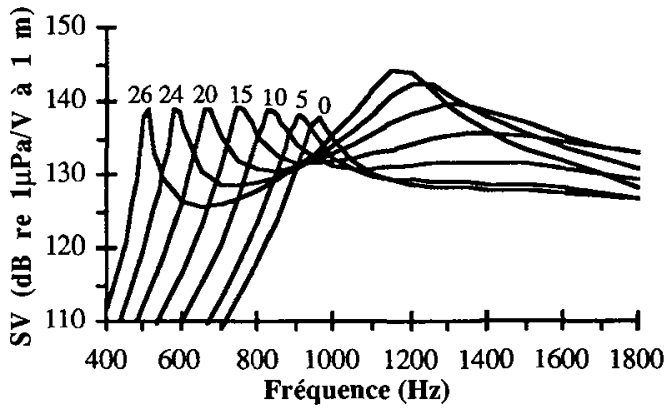

Fig. 8 : réponse radiale avec baffles différents $(0$ à $26 \mathrm{~cm})$

Les mesures et les modélisations prouvent ainsi que la première résonance est une résonance du moteur où le pavillon joue le rôle d'excitateur entraînant le fluide de la cavité (l'étude du champ de pressions dans la cavité indique un fort gradient, la pression étant maximale sur la face arrière du pavillon, puis décroissant régulièrement vers l'évent). Cette résonance décroît en fréquence avec la longueur du baffle car l'importante masse d'eau en mouvement dans la cavité modifie l'impédance de rayonnement du pavillon. Ce résultat confirme les calculs d'optimisation de la cavité d'un Janus-Helmholtz [4].

\subsection{Deuxième résonance et résonance de Helmholtz}

Les résultats précédents ont montrés que l'effet de la compliance de la cavité était très important sur la deuxième résonance, qui est d'autant plus basse en fréquence que la cavité est élastique. L'étude de la nature de cette résonance (en utilisant en parallèle les modélisations éléments-finis et schémas équivalents) nous apprend d'une part que la masse dynamique du moteur joue un rôle important à ces fréquences et, d'autre part, que l'accélération du pavillon est élevée. De plus, le calcul de la résonance de Helmholtz (différent des valeurs mesurées de la deuxième résonance), le peu d'effet des dimensions du col du résonateur et l'étude du champ de pressions (maximal sur la face avant des pavillons) tendent à prouver que cette résonance est une deuxième résonance du moteur, fortement influencée par la compliance de la cavité.

Pour vérifier l'existence d'une résonance de Helmholtz, l'étude de la résonance du résonateur a révélé qu'elle se produisait à une fréquence correspondant exactement au minimum situé entre les deux résonances. L'importante influence de la taille de l'évent et la pression constante dans la cavité confirment la présence d'une résonance de Helmholtz entre les deux résonances du moteur.

\section{CONCLUSION ET APPLICATION A LA TOMOGRAPHIE ACOUSTIQUE}

L'étude menée sur le fonctionnement du Janus-Helmholtz, dans une bande de fréquences inférieure à 2 $\mathrm{kHz}$, a montré que l'interprétation généralement faite des deux résonances était inexacte. La connaissance des paramètres influençant chaque résonance a permis par la suite de faire chuter la première résonance tout en conservant un bon couplage entre les deux résonances. Il a ainsi été possible de réaliser un JanusHelmholtz $300 \mathrm{~Hz}$ (fréquences divisées par deux par rapport au transducteur standard) en modifiant les dimensions du baffle et des pavillons [5]. Les objectifs de grande portée pourront alors être atteints à l'aide de ce transducteur TBF dont les dimensions sont faibles en regard des fréquences obtenues (longueur maximale de $55 \mathrm{~cm}$, soit $1 / 10^{\mathrm{e}}$ de la longueur d'onde acoustique à la résonance).

\section{REFERENCES :}

[1] W. Munk, C. Wunsch : "Ocean acoustic tomography : a scheme for large scale monitoring". Deep Sea Res., vol. 26, pp 123 - 161 (1978).

[2] Y. Le Gall, D. Boucher, X. Lurton : "Depth-unlimited versions of the Janus-Helmholtz : a new interpretation of working principles - Some experimental results", Proceedings of U.D.T., Cannes, France, 1993.

[3] J.N. Decarpigny, B. Hamonic : "General Introduction to ATILA". Proceedings of the workshop, Toulon, France, 1990.

[4] R. Bossut, B. Hamonic : "Optimisation de la cavité d'un Janus-Helmholtz 1100", Rapport ISEN, 1992.

[5] Y. Le Gall, D. Boucher, X. Lurton, A.M. Bruneau : "A $300 \mathrm{~Hz}$ Janus-Helmholtz transducer for Ocean Acoustic tomography", Proceedings of OCEANS'93, Victoria, Canada, 1993. 